\title{
A complete description of the magnetic ground state in spinel vanadates
}

\author{
Jyoti Krishna, ${ }^{1}$ N. Singh, ${ }^{2}$ S. Shallcross,${ }^{3}$ J. K. Dewhurst,${ }^{4}$ E. K. U. Gross,${ }^{4}$ T. Maitra, ${ }^{1}$ and S. Sharma ${ }^{2}$ \\ ${ }^{1}$ Department of Physics, Indian Institute of Technology Roorkee, Roorkee - 247667, Uttarakhand, India \\ ${ }^{2}$ Max Born Institute for Nonlinear Optics and Short Pulse Spectroscopy, Max-Born-Strasse 2A, 12489 Berlin, Germany \\ ${ }^{3}$ Lehrstuhl für Theoretische Festkörperphysik, Staudstr. 7-B2, 91058 Erlangen, Germany \\ ${ }^{4}$ Max-Planck-Institut für Mikrostrukturphysik, Weinberg 2, D-06120 Halle, Germany*
}

(Dated: March 18, 2019)

\begin{abstract}
Capturing the non-collinear magnetic ground state of the spinel vanadates $\mathrm{AV}_{2} \mathrm{O}_{4}(\mathrm{~A}=\mathrm{Mn}, \mathrm{Fe}$ and $\mathrm{Co}$ ) remains an outstanding challenge for state-of-the-art ab-initio methods. We demonstrate that both the non-collinear spin texture, as well as the magnitude of local moments, are captured by a single value of the on-site Hubbard $U$ of $2.7 \mathrm{eV}$ in conjunction with the local spin density approximation $(\mathrm{LSDA}+U)$, provided the source term (i.e., magnetic monopole term) is removed from the exchange-correlation magnetic field $\mathbf{B}_{X C}$. We further demonstrate that the magnetic monopole structure in $\mathbf{B}_{X C}$ is highly sensitive to the value of $U$, to the extent that the interplay between on-site localization and local moment magnitude is qualitatively different depending on whether the source term is removed or not. This suggests that in treating strongly correlated magnetic materials within the $\mathrm{LSDA}+U$ formalism, subtraction of the unphysical magnetic monopole term from the exchange-correlation magnetic field is essential to correctly treat the magnetic ground state.
\end{abstract}

The strongly correlated electron systems (SCES) derive their richness from competing and coexisting multiple long range orders (LROs) such as charge, magnetic, orbital order [1]. A strong interplay among various degrees of freedom (e.g. charge, spin, orbital and lattice) in these materials provides a perfect platform for both basic and applied physics questions [2]. The family of spinel vanadates $\left(\mathrm{AV}_{2} \mathrm{O}_{4}\right)$ belongs to such a class of materials where strong correlation, complex spin texture, and geometric frustration of the underlying lattice work in tandem [3, 4], and this richness of physics has attracted the sustained attention of the condensed matter and materials science community [4-9].

However, as several invesigations have made clear, the modern day theoretical method of choice, namely density functional theory (DFT) [10, 11], fails to describe spinel vanadates in two crucial ways. Firstly, in experiment the moment on $\mathrm{V}$ atoms is much lower (e.g. 1.3 $\mu_{B}$ in $\mathrm{MnV}_{2} \mathrm{O}_{4}$ [5] to $0.65 \mu_{B}$ in $\mathrm{ZnV}_{2} \mathrm{O}_{4}$ [12]) than the DFT values $[6,13-15]$ with difference between the two as high as $60 \%$. The reasons behind this large reduction in $\mathrm{V}$ moment remains contested with speculations including spin frustration, quantum fluctuations and spin-orbit interaction effects. Secondly, the experimentally observed ground state magnetic structure is a complex spin texture i.e. a non-collinear arrangement of $\mathrm{V}$ spins (with a large angle between $\mathrm{A}$ and $\mathrm{V}$ spins), while DFT predicts a collinear ferrimagnetic ground-state [6, 13-15, 29-31]. This incorrect DFT ground-state entails that the interesting physics of magnetic phase transitions in these materials stays beyond any ab-initio description.

In the present work, with an example of three spinel vanadates $\left(\mathrm{FeV}_{2} \mathrm{O}_{4}, \mathrm{MnV}_{2} \mathrm{O}_{4}\right.$ and $\left.\mathrm{CoV}_{2} \mathrm{O}_{4}\right)$, we probe the reason behind the failure of DFT, which is otherwise an excellent theory for ab-initio description of complex magnets. DFT is in principle an exact theory, but in practice requires an approximation for the so called exchange-correlation (XC) functional. In the present work we demonstrate that this approximation lies at the heart of the failure to capture the magnetic structure; local spin density approximation (LSDA)[16] and generalized gradient approximation (GGA)[17] like functionals generate magnetic fields, $\left(\mathbf{B}_{X C}\right)$, which have a large source term leading to magnetic mono-poles (i.e. $\left.\boldsymbol{\nabla} \cdot \mathbf{B}_{X C} \neq 0\right)$. The presence of this source term in turn leads to large discrepancy in calculated magnetic ground-state and experimental data. Removal of this source-term, by using recently developed source-free XC functional[18], reproduces the experimentally observed non-collinear magnetic state (both in terms of the canting angle as well as the magnitude of $\mathrm{V}$ moments) in all the three spinel vanadates. Furthermore, we also demonstrate that on-site Coulomb correlation $U$, used to treat strong-correlations in materials via $\mathrm{LSDA}+U$ like approach, has the effect of increasing this unphysical sourceterm, removal of which makes LSDA/GGA $+U$ method highly accurate for the materials under consideration.

The ground state DFT calculations were carried out within full potential linearized augmented plane wave (LAPW) as implemented in the ELK code [19]. All calculations were performed in the presence of spin-orbit coupling term in the Hamiltonian. A k-point grid of $8 \times 8 \times 6$ was used. The exchange-correlation effect were treated using the local spin density approximation (LSDA) and LSDA $+U$ functionals. A fully unconstrained minimization was performed; a random magnetic field was applied to break the symmetry and subsequently reduced to zero over self-consistent cycle. In this way the selfconsistent magnetization is not biased by the initial guess of the magnetization density, which is treated as a unconstrained vector field. The structural parameters of $(\mathrm{Mn}$, $\mathrm{Fe}, \mathrm{Co}) \mathrm{V}_{2} \mathrm{O}_{4}$ were taken from experiments [20, 21]. 


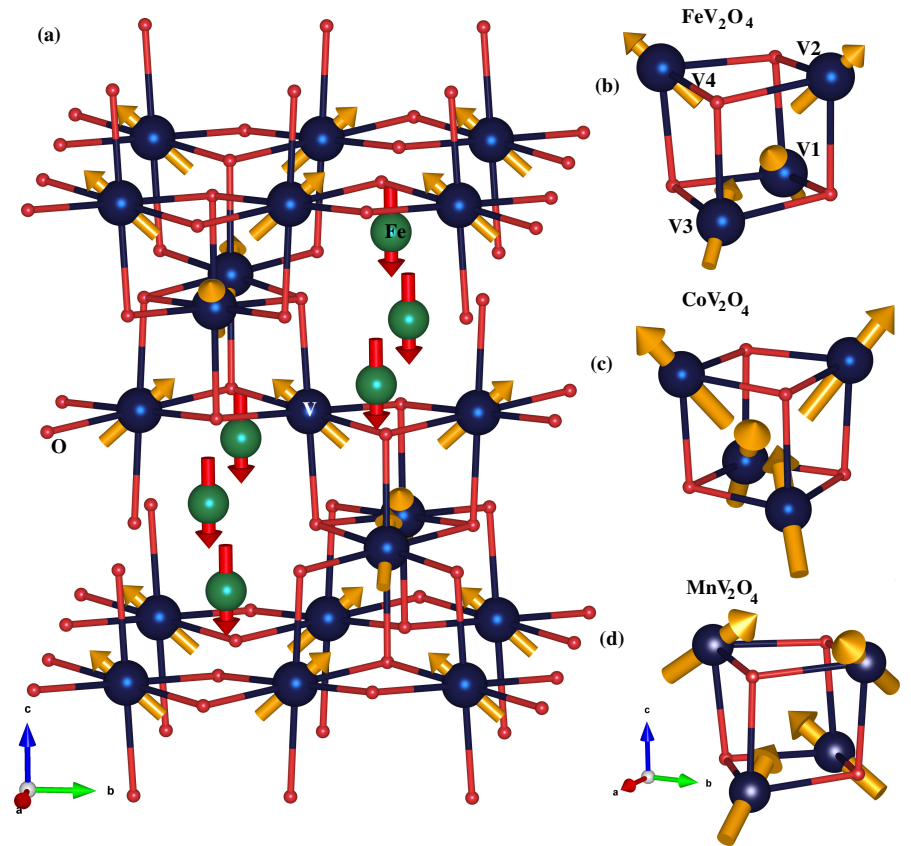

FIG. 1. The non-collinear magnetic structure obtained using $\mathrm{LSDA}_{S F}+\mathrm{U}$ with $\mathrm{U}=2.7 \mathrm{eV}$ shown (a) within the unit cell for $\mathrm{FeV}_{2} \mathrm{O}_{4}$; within $\mathrm{V}_{4} \mathrm{O}_{4}$ cube for (b) $\mathrm{FeV}_{2} \mathrm{O}_{4}$, (c) $\mathrm{CoV}_{2} \mathrm{O}_{4}$ and (d) $\mathrm{MnV}_{2} \mathrm{O}_{4}$.

Spin-texture: In the insulating non-collinear magnetic ground-state observed experimentally all three materials $[5,21,23,25,26]$ possess the $\mathrm{A}(\mathrm{A}=\mathrm{Fe}, \mathrm{Co}, \mathrm{Mn})$ site spin moments aligned along the $c$-axis, while the $\mathrm{V}$ moments significantly cant away from the $c$-axis (by an angle of up to[5] 65 ${ }^{\circ}$ ). In $\mathrm{FeV}_{2} \mathrm{O}_{4}$ and $\mathrm{CoV}_{2} \mathrm{O}_{4}$, the $\mathrm{V}$-moments form a structure known as "two-in-two-out" structure in each $\mathrm{V}_{4} \mathrm{O}_{4}$ cube[23, 27] whereas in $\mathrm{MnV}_{2} \mathrm{O}_{4}$ the observed structure is somewhat more complex[5].

In contradiction to experiments, DFT calculations using LSDA show a metallic collinear ground-state. Since vanadates are strongly correlated insulators [4, 28] adding an on-site Coulomb repulsion by using the LSDA $+U$ method, as expected, opens a gap. However, the magnetic ground-state stays collinear ferrimagnet $[6,13-$ 15, 29-31], a situation that cannot be improved by changing the functional from LSDA $+U$ to $\mathrm{GGA}+U$ or metaGGA. Adding spin-orbit coupling to the Hamiltonian introduces a weak non-collinearity.

In order to understand the reason behind this profound discrepancy between theory and experiments we examine the approximate $\mathrm{XC}$ functionals used. It has been shown before that for materials such as Fe Pnictides the incorrect magnetic ground-state can be attributed to the unphysical source term in the LSDA (and GGA) XC magnetic fields, removal of which, via source-free XC functional, results in agreement with experiments[18]. In the present case the problem is more complex in that not only the magnitude (as in the case of Fe-Pnictides), but also the direction of the local moments obtained using $\mathrm{LSDA}+U$ functional are incorrect. Whether the source term in LSDA $+U$ magnetic field is also responsible for the incorrect spin texture in these materials remains to be seen.

We employ the source-free LSDA $+U$ functional (hereafter denoted by $\left.\operatorname{LSDA}_{S F}+U\right)$ to perform a fully unconstrained optimization of magnetization density (both the direction and magnitude). In agreement with experiments we find a non-collinear magnetic ground state for all three compounds. Most importantly, a single value of $U$ (i.e. $U=2.7 \mathrm{eV}$ acting on the $\mathrm{V}$-atoms) is required to reproduce the experimentally observed diverse noncollinear magnetic ground states of all the three compounds: both the "two-in-two-out" [23, 27] spin arrangements of $\mathrm{FeV}_{2} \mathrm{O}_{4}$ and $\mathrm{CoV}_{2} \mathrm{O}_{4}$ (Fig. 1a and 1b) and the complex spin-texture in $\mathrm{MnV}_{2} \mathrm{O}_{4}[23,27]$ (Fig. 1c) are perfectly captured.

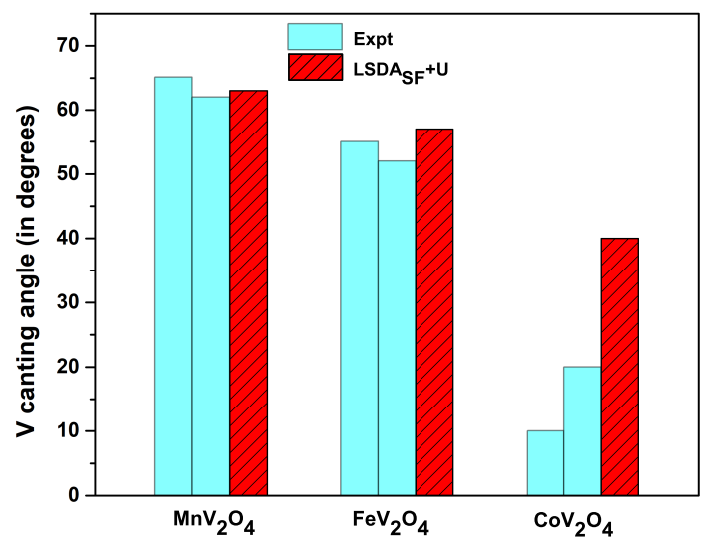

FIG. 2. Canting angle of $\mathrm{V}$ moments with respect to the $c$ axis calculated with source-free LSDA $+U$ (red) and compared with the corresponding experimental values [5, 21, 23, 26, 32, 33] (cyan) for $\mathrm{MnV}_{2} \mathrm{O}_{4}, \mathrm{FeV}_{2} \mathrm{O}_{4}$ and $\mathrm{CoV}_{2} \mathrm{O}_{4}$. The on-site Coulomb repulsion $U$ is equal to $2.7 \mathrm{eV}$ for all materials. Note that employment of the standard LSDA functional results in a grossly wrong value of the canting angle of $0^{\circ}$ which increases to $9^{\circ}$ upon inclusion of the spin-orbit coupling term.

As for the value of the angle between the $\mathrm{V}$ and $\mathrm{A}$ site spins, consistent with the experimental observations our results show that the $\mathrm{A}$ site moment is collinear with the $c$-axis in presence or absence of the source term in the functional. On the other hand, the canting angle of the $\mathrm{V}$ spins is highly functional dependent in that LSDA $+U$ functional, in presence of spin-orbit coupling, leads to a small canting angle of $19^{\circ}$. These results are unlike experiments which show that the canting angle is much smaller in $\mathrm{CoV}_{2} \mathrm{O}_{4}$ than in $\mathrm{MnV}_{2} \mathrm{O}_{4}$ or $\mathrm{FeV}_{2} \mathrm{O}_{4}$. Removal of the source term from this functional has a dramatic effect on the canting angle (see Fig. 2); for $\mathrm{MnV}_{2} \mathrm{O}_{4}$ and 
TABLE I. Magnetic moments (in $\mu_{B}$ ) per A-site atom.

\begin{tabular}{||c|c|c|c|c|c||}
\hline Vanadate & Expt. & LSDA & $L S D A_{S F}$ & $\begin{array}{c}\text { LSDA+U } \\
(U=\mathbf{2 . 7} \mathbf{~ e V})\end{array}$ & $\begin{array}{c}L S D A_{S F}+U \\
(U=\mathbf{2 . 7} \mathbf{~ e V})\end{array}$ \\
\hline \hline $\mathrm{MnV}_{2} \mathrm{O}_{4}$ & $4.2[5], 4.11[22]$ & 4.02 & 3.98 & 4.11 & 4.10 \\
\hline $\mathrm{FeV}_{2} \mathrm{O}_{4}$ & $4[23]$ & 3.22 & 3.15 & 3.42 & 3.44 \\
\hline $\mathrm{CoV}_{2} \mathrm{O}_{4}$ & $2.46[24]$ & 2 & 2.08 & 2.45 & 2.41 \\
\hline \hline
\end{tabular}

$\mathrm{FeV}_{2} \mathrm{O}_{4}$ the agreement with experiments is excellent but for $\mathrm{CoV}_{2} \mathrm{O}_{4}$ the results overshoot slightly. However, consistent with the experimental trend $[5,23,26,32,33]$ we find that the canting angle is smaller in $\mathrm{CoV}_{2} \mathrm{O}_{4}$ than in $\mathrm{MnV}_{2} \mathrm{O}_{4}$ or $\mathrm{FeV}_{2} \mathrm{O}_{4}$.

Magnitude of the moment: As discussed in the introduction, experimental measurements via neutron diffraction [5, 23, 27], XMCD [24] etc. on spinel vanadates report a small moment on the $\mathrm{V}$ atoms in all these compounds; $1.3 \mu_{B}$ in $\mathrm{MnV}_{2} \mathrm{O}_{4}, 0.85 \mu_{B}$ in $\mathrm{FeV}_{2} \mathrm{O}_{4}, 0.9 \mu_{B}$ in $\mathrm{CoV}_{2} \mathrm{O}_{4}$. This moment is much smaller than $2 \mu_{B}$, the expected value for a $\mathrm{V}^{3+}$ state. Furthermore, XMCD measurements performed on $\mathrm{MnV}_{2} \mathrm{O}_{4}$ and $\mathrm{FeV}_{2} \mathrm{O}_{4}$ reveal a very small value of the orbital moment [22] indicating the $\mathrm{V}$ moment is primarily spin in character [34], and so the cancellation of the spin-moment by the orbital moment cannot be the reason behind this reduction in the moment. As for the moment on the A-site is concerned, all experiments report a large moment [5, 22-24].

In contraction to these experiments, and in agreement with previous ab-initio work[6,31], we find that DFT calculations performed using the LSDA and LSDA $+U$ functionals show a large moment on $\mathrm{V}$ atoms with percentage deviation of up to $40 \%$ from experiment (see Fig. 3a). Interestingly, there does not exist a value of $U$ for which the correct moment on the $\mathrm{V}$ atoms can be obtained (see Fig. 3b). Use of $\operatorname{LSDA}_{S F}$ and $\mathrm{LSDA}_{S F}+U$ with $U=2.7 \mathrm{eV}$, the value of $U$ that gives correct spin texture, remarkably, also leads to the value of the $\mathrm{V}$ moment in close agreement with experiments with the worst error only a $2 \%$ deviation (see Fig. 3a). The moment on the A site is well described by LSDA/LSDA $+U$ and their source-free counterparts (see Table I). Thus the source free LSDA $+U$ functional provides complete description of the ground-state of all three spinel vanadates with a single value of $U$.

A study of the magnitude of the $\mathrm{V}$ moment as a function of $U$ leads to a striking observation; increase of $U$ within LSDA $+U$ functional generates, as expected, increased on-site localization of charge and an increased local moment on the $\mathrm{V}$ atoms (see Fig. 3(b)). A consequence of this is that there does not exist a value of $U$ for which the correct value of $\mathrm{V}$ moment is obtained. However, the $\mathrm{V}$ moment calculated by excluding the source term from the LSDA $+U$ functional shows exactly the opposite trend: the $\mathrm{V}$ moment decreases with $U$ (see Fig. $3(\mathrm{c})$ ). This is a counter intuitive yet explainable trend- as
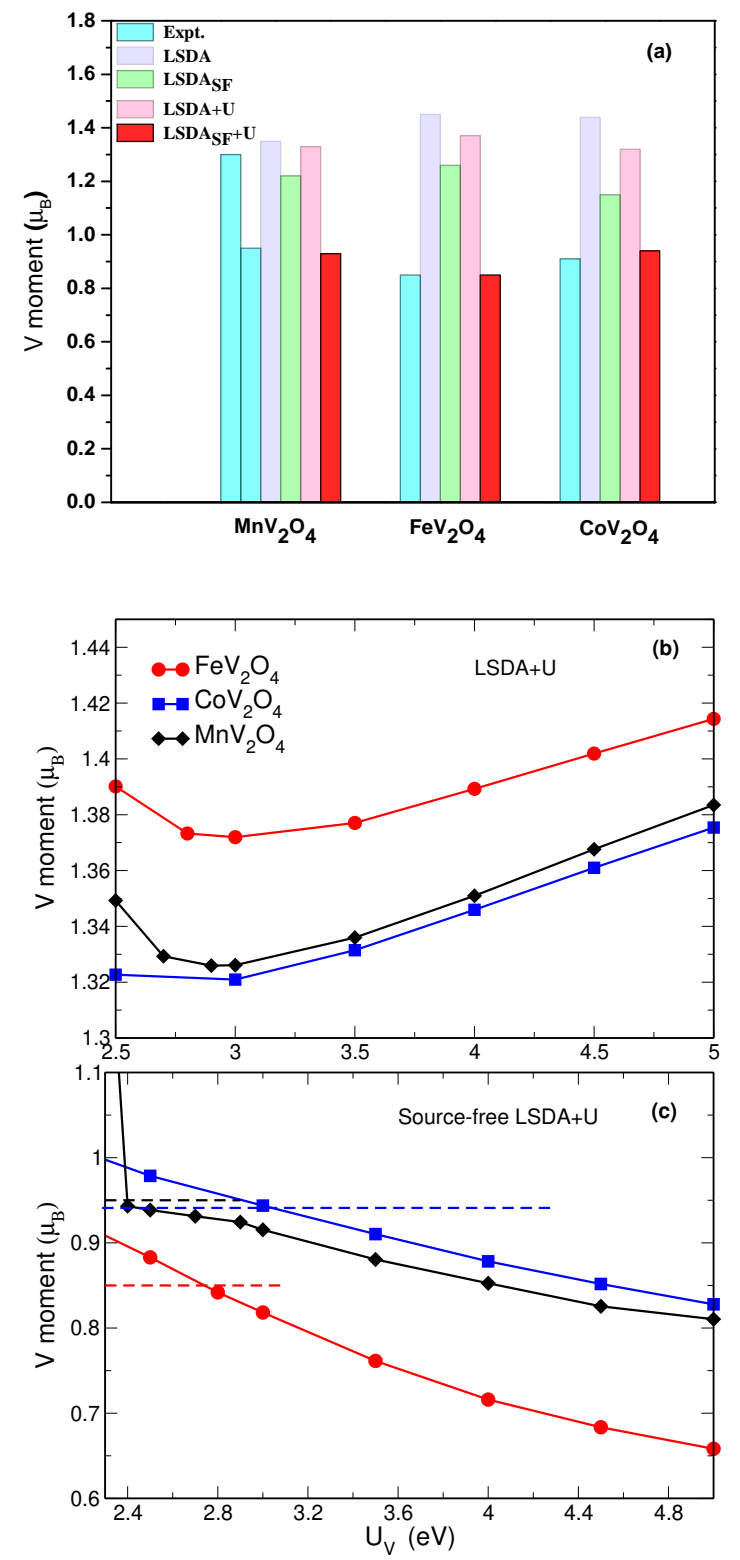

FIG. 3. (a) V magnetic moment calculated using LSDA (light blue), $\operatorname{LSDA}_{S F}$ (green), LSDA $+U$ (pink) and LSDA $+U_{S F}$ (red) compared with corresponding experimental (cyan) values [5, 21, 23, 24, 27] for $\mathrm{MnV}_{2} \mathrm{O}_{4}, \mathrm{FeV}_{2} \mathrm{O}_{4}$ and $\mathrm{CoV}_{2} \mathrm{O}_{4}$. The on-site Hubbard $U$ parameter is set equal to $2.7 \mathrm{eV}$ for all materials. Variation of total spin magnetic moment of $\mathrm{V}$ as a function of this on-site Hubbard $U$ using (b) $\mathrm{LSDA}+U$ and (c) source-free LSDA $+U \mathrm{~V}\left(m_{V}\right)$ functionals.

the value of $U$ increases the source term in the XC magnetic field also increases. The removal of this source term then has a significant effect on the magnetization density leading to a decrease in the $\mathrm{V}$ moment as a function of increasing $U$. This suggests that in treating the magnetic ground state of strongly correlated materials within the LSDA $+U$ framework, varying $U$ can in an uncontrolled 
way also alter the unphysical source term in $\mathbf{B}_{X C}$. Thus it would appear imperative in treating strong correlated magnetic ground states that $\mathbf{B}_{X C}$ should be source free.

In conclusion, we have investigated the magnetic ground-state for spinel vanadates $\mathrm{AV}_{2} \mathrm{O}_{4}(\mathrm{~A}=\mathrm{Mn}, \mathrm{Fe}$ and $\mathrm{Co}$ ) using density functional theory. In doing so we find that the well known failure of all traditional $\mathrm{XC}$ functionals (LSDA, GGA, LSDA $+U$, meta GGA) to reproduce the experimentally observed magnitude of local moments and non-collinear spin texture arises from the presence of a large source term in the magnetic field generated by these functionals. Most strikingly, we find that this source-term increases on increasing the value of $U$. Removing this unphysical source-term from LSDA $+U$ functional we find results in a perfect description of the ground-state magnetism of these materials. Most importantly we find that for all three materials we needed the same value of $U$ for this correct description. This is a great improvement over traditional LSDA $+U$ approach where there does not exist a single value of $U$ which gives the correct ground-state for these materials.

This work is supported by DST-DAAD project (grant no INT/FRG/DAAD/P-16/2018) funded by DST(India) and DAAD (Germany). JK would like to acknowledge MHRD(INDIA) research fellowship. Sharma expresses gratitude to the DFG for funding through TRR227 (project A04).

* dewhurst@mpi-halle.mpg.de

[1] E. Dagotto, Science 309, 257 (2005).

[2] Y. Tokura, Physics Today 56, 50 (2003).

[3] P. G Radaelli, New Journal of Physics 7, 53 (2005).

[4] S.-H. Lee, H. Takagi, D. Louca, M. Matsuda, S. Ji, H. Ueda, Y. Ueda, T. Katsufuji, J.-H. Chung, S. Park, S.-W. Cheong, and C. Broholm, Journal of the Physical Society of Japan 79, 011004 (2010).

[5] V. O. Garlea, R. Jin, D. Mandrus, B. Roessli, Q. Huang, M. Miller, A. J. Schultz, and S. E. Nagler, Physical Review Letters 100, 066404 (2008).

[6] S. Sarkar, T. Maitra, R. Valenti, and T. Saha-Dasgupta, Physical Review Letters 102, 216405 (2009).

[7] Q. Zhang, K. Singh, F. Guillou, C. Simon, Y. Breard, V. Caignaert, and V. Hardy, Physical Review B 85, 054405 (2012).

[8] E. M. Wheeler, B. Lake, A. T. M. Nazmul Islam, M. Reehuis, P. Steffens, T. Guidi, and A. H. Hill, Physical Review B 82, 140406(R) (2010).

[9] T. Suzuki, M. Katsumura, K. Taniguchi, T. Arima, and T. Katsufuji, Physical Review Letters 98, 127203 (2007).

[10] P. Hohenberg and W. Kohn, Physical Review 136, B864 (1964).

[11] W. Kohn and L. J. Sham, Physical Review 140, A1133 (1965).

[12] M. Reehuis, A. Krimmel, N. Büttgen, A. Loidl, and A. Prokofiev, The European Physical Journal B 35, 311 (2003).

[13] R. Nanguneri, and S. Y. Savrasov, Physical Review B
86, 085138 (2012).

[14] S. Sarkar, and T. Saha-Dasgupta, Physical Review B 84, 235112 (2011).

[15] T. Maitra, and R. Valenti, Physical Review Letters 99, 126401 (2007).

[16] J. P. Perdew and Y. Wang, Physical Review B 45, 13244 (1992)

[17] J. P. Perdew, A. Ruzsinszky, G. I. Csonka, O. A. Vydrov, G. E.Scuseria, L. A. Constantin, X. Zhou, and K. Burke, Physical Review Letters 100, 136406 (2008).

[18] S. Sharma, E. K. U. Gross, A. Sanna, and J. K. Dewhurst, Journal of Chemical Theory and Computation 14, 1247 (2018).

[19] http://elk.sourceforge.net

[20] Y. Nii, H. Sagayama, T. Arima, S. Aoyagi, R. Sakai, S. Maki, E. Nishibori, H. Sawa, K. Sugimoto, H. Ohsumi, and M. Takata, Physical Review B 86, 125142 (2012).

[21] H. Ishibashi, S. Shimono, K. Tomiyasu, S. Lee, S. Kawaguchi, H. Iwane, H. Nakao, S. Torii, T. Kamiyama, and Y. Kubota, Physical Review B 96, 144424 (2017).

[22] J. Okabayashi, S. Miyasaka, K. Hemmi, K. Tanaka, S. Tajima, H. Wadati, A. Tanaka, Y. Takagi, and T. Yokoyama, Journal of the Physical Society of Japan 84, 104703 (2015).

[23] G. J. MacDougall, V. O. Garlea, A. A. Aczel, H. D. Zhou, and S. E. Nagler, Physical Review B 86, 060414(R) (2012).

[24] Y. Nonaka, G. Shibata, R. Koborinai, K. Ishigami, S. Sakamoto, K. Ikeda, Z. Chi, T. Koide, A. Tanaka, T. Katsufuji, and A. Fujimori, Physical Review B 97, 205126 (2018).

[25] J. Chung, Y. Song, J. Kim, T. Suzuki, T. Katsufuji, M. Matsuda, and S. Lee, Physical Review B 88, 094430 (2013).

[26] R. Koborinai, S. E. Dissanayake, M. Reehuis, M. Matsuda, S.-H. Lee, and T. Katsufuji, Physical Review Letters 116, 037201 (2016).

[27] J. H. Lee, J. Ma, S. E. Hahn, H. B. Cao, M. Lee, T. Hong, H.-J. Lee, M. S. Yeom, S. Okamoto, H. D. Zhou, M. Matsuda and R. S. Fishman, Scientific Reports 7, 17129 (2017).

[28] S. Blanco-Canosa, F. Rivadulla, V. Pardo, D. Baldomir, J.-S. Zhou, M. Garcia-Hernandez, M. A. Lopez-Quintela, J. Rivas, and J. B. Goodenough, Physical Review Letters 99, 187201 (2007).

[29] R. Kaur, T. Maitra, and T. Nautiyal, Journal of Physics: Condensed Matter 26, 045505 (2014).

[30] V. Pardo, S. Blanco-Canosa, F. Rivadulla, D. I. Khomskii, D. Baldomir, H. Wu, and J. Rivas, Physical Review Letter 101, 256403 (2008).

[31] D. Dey, T. Maitra and A. Taraphder, Physical Review B 93, 195133 (2016).

[32] L. Zhang, H. Han, Z. Qu, J. Fan, L. Ling, C. Zhang, L. Pi, and Y. Zhang, Journal of Applied Physics 115, 233910 (2014).

[33] S. Nakamura, Y. Kobayashi, S. Kitao, and M. Seto, Physica B: Condensed Matter 536, 620 (2018).

[34] K. Matsuura, H. Sagayama, Y. Nii, N. D. Khanh, N. Abe, and T. Arima, Physical Review B 92, 035133 (2015).

[35] Z.-Y. Li, X. Li, J.-G. Cheng, L. G. Marshall, X.-Y. Li, A. M. Santos, W.-G. Yang, J. J. Wu, J.-F. Lin, G. Henkelman, T. Okada, Y. Uwatoko, H. B. Cao, H. D. Zhou, J. B. Goodenough, and J.-S. Zhou, Physical Review B 94, 165159 (2016). 